Aim of the study: Tumour endothelial cells have been proven to have molecular markers distinct from normal endothelial cells. These specific molecular markers allow for targeting of the tumour vasculature with specific pharmacological vehicles to direct diagnostic or therapeutic modalities at the endothelial cells. By performing a phage display-based screening, this study aimed to identify a certain short peptide that could specifically bind to osteosarcoma vasculature.

Material and methods: We performed in vivo screening in the murine models of osteosarcoma with annular Ph.D.C7C library in the present study. To explore the in vivo binding specificity of the retrieved peptide, we conjugated the peptide with fluorescein isothiocyanate (FITC) and injected it intravenously into osteosarcoma-bearing BALB-c mice.

Results: CTKPDKGYC was the dominant sequence isolated from in vivo screening and was named as NF-1. Fluorescence staining found that FITC-NF-1 peptide could be specifically homed to osteosarcoma vasculature while being almost undetectable in the heart, brain, lung and liver. Simultaneously, a small amount of fluorescence could also be detected in the renal glomerulus and renal tubule but not in renal vascular endothelium, indicating that FITC-NF-1 peptide might be excreted mainly through the renal-urinary route.

Conclusions: Our data suggest that, with high binding specificity to osteosarcoma vasculature, peptide NF-1 may have potential value in early diagnosis or targeted therapy for osteosarcoma.

Key words: osteosarcoma (OS), phage display, peptide, angiogenesis.

Contemp Oncol (Pozn) 2014; 18 (3): 165-170 DOI: $10.5114 /$ wo.2014.41384

\section{Targeting osteosarcoma vasculature with peptide obtained by phage display}

\author{
Mingqiang Guan*, Jian Wang*, Lanbo Yang, Zandong Zhao, Kun Lu, \\ Liang Zhao, Jun Xiao, Zhihan Li, Zhanjun Shi
}

Department of Orthopaedic Surgery, Nanfang Hospital, Southern Medical University, Guangzhou, Guangdong Province, PR China

"These authors contributed equally to this work.

\section{Introduction}

Osteosarcoma is the most common primary malignant bone tumour in children and adolescents [1]. The standard treatment strategy of osteosarcoma at present is the complete removal of the tumour by wide excision and aggressive chemotherapy [2]. Although this strategy has significantly improved the survival rate from $11 \%$ to $70 \%$ [3], it seems that improvements in survival have reached a plateau in recent years [4]. Metastases, chemoresistance and serious side effects remain the major reasons for the failure of osteosarcoma treatment [5]. Thus, novel agents that can enhance the local drug concentration in the tumour while reducing undesirable side effects are needed in order to achieve further improvement in the survival rate. Tumour angiogenesis is known to be an indispensable process during tumour growth and metastasis [6]. Accumulative evidence indicates that tumour endothelial cells possess molecular markers distinct from normal endothelial cells [7-9]. Specific molecular markers would allow targeting of the tumour vasculature [10-12]. This creates the possibility of developing specific pharmacological tools to direct diagnostic or therapeutic modalities at the endothelial cells $[13,14]$. Because of several clinical advantages, small peptides obtained by phage display technology seem to be promising pharmacological tools. For instance, small peptides display good tissue penetrating ability due to their small molecular weight (average less than 50 amino acids), low immunogenicity, high affinity to targets, acceptable stability and integrity in vivo and easy manipulation for synthesis and conjugation with other agents [15-17]. To date, several functionally relevant homing peptides have been identified in tumour vasculature. They may be used as promising vehicles to transport more selectively diagnostic or therapeutic cargo to tumour endothelial cells [18-21]. In a recent study [22], the authors successfully identified a new peptide by in vitro phage screening technology using human osteosarcoma cells but not osteosarcoma vasculature as the selected target. When the peptide was labeled with fluorescein isothiocyanate, successful noninvasive PET imaging of osteosarcoma tumours was possible in nude mice, indicating peptide imaging as a promising strategy for early detection of osteosarcoma.

In the present study, we firstly established osteosarcoma xenograft with osteosarcoma cell line Saos-2, which was obtained from the American Type Culture Collection (ATCC), primarily cultured from an 11-year-old female Caucasian. We then performed in vivo phage screening process using tumour vasculature as the selected target. The in vivo binding specificity of the peptide obtained from the phage display was then confirmed under laser scanning microscope (LSCM) when it was conjugated FITC. 


\section{Material and methods}

\section{Materials}

The disulphide-constrained (seven amino acids with a flanking cysteine residue at both ends of the peptide) cyclic M13 phage display library (Ph.D.C7C system; New England Biolabs, Hitchin, UK) was used. Dulbecco's modified Eagle's medium (DMEM) and foetal calf serum (FCS) were purchased from Hyclone (Thermo Scientific, USA).

\section{Cell culture}

The human osteosarcoma cell line Saos-2 was obtained from the American Type Culture Collection (ATCC) and maintained in DMEM supplemented with $10 \%$ FCS $(\mathrm{V} / \mathrm{V})$ in a $5 \% \mathrm{CO}_{2}$ atmosphere.

\section{Osteosarcoma xenograft}

The osteosarcoma xenograft was established by subcutaneous injection of Saos- 2 cells $\left(1 \times 10^{7}\right.$ cells in $200 \mu \mathrm{l}$ DMEM) into the front flank of BALB-c mice (Southern Medical University, China) at 4 weeks of age. All animal experiments were approved by the ethics committee of Southern Medical University.

\section{In vivo panning}

We continued with the in vivo experiment when the tumour volume reached at least $80 \mathrm{~mm}^{3}$ (about 9 weeks after cell injection). The phage library $\left[1 \times 10^{11}\right.$ plaque-forming units (PFU) in $200 \mu \mathrm{lPBS}$ ] was injected into the tail vein of anaesthetised animals. After 10 minutes, the mice were perfused via the left ventricle with $20 \mathrm{ml}$ of DMEM to ensure phage clearance from the blood. The inferior vena cava was cut for the outlet. Tumours and control organs (heart, brain, lung, liver and kidney) were extracted, weighed and quick-frozen in liquid nitrogen for 5 minutes and were then ground in DMEM with protease inhibitors (1 mM phenylmethylsulfonyl fluoride, $20 \mathrm{mg} / \mathrm{ml}$ aprotinin, and $1 \mathrm{mg} / \mathrm{ml}$ leupeptin) and $0.5 \%$ bovine serum albumin (BSA) and washed five times by centrifugation at 6000 rpm. Phages were eluted from the tissues with $1.6 \mathrm{ml}$ of $0.1 \mathrm{M}$ glycine, $\mathrm{pH} 2.0$, and after 10 minutes of incubation, were neutralised with $36 \mu$ of $2 \mathrm{M}$ Tris base. To determine and compare the number of phages in the eluate from tumours and control organs, in each round of selection, $100 \mu \mathrm{l}$ of the eluate was added with the Escherichia coli host ER2738 into melted Luria-Bertani (LB) agar tops, which were then plated onto isopropyl- $\beta$-D-thiogalactopyranoside (IPTG)/X-Gal LB agar plates. After overnight incubation at $37^{\circ} \mathrm{C}$, the peptide phages, appearing as blue plaques, were counted and the yield of phage localising to each individual tissue was determined. The residual eluate from the tumour was amplified and used as the input phage for the next round of in vivo selection. Three further cycles of in vivo selection were performed to enrich for specificity to osteosarcoma vasculature. The eluate of the last cycle from the tumour was re-injected into the tail vein of normal non-tumour-bearing BALB-c mice for 10 minutes to clear phages that bound to the normal vasculature and other non-tumour antigens.

\section{Elimination of the phages without inserted sequences}

After subtractive screening with normal non-tumourbearing BALB-c mice, 72 individual clones were picked at random and their DNA was amplified by PCR. Then the clones without inserted sequences were identified and eliminated by agarose gel electrophoresis with the PCR product, since the DNA with inserted sequences was about 300 bp with lower speed in agarose gel electrophoresis, while the one without inserted sequences was only 258 bp with higher speed.

\section{Sequence analysis}

In total 61 phage clones with inserted sequences were sequenced (Beijing Huada Gene Corp, China). The primer used for sequencing was 5'-HOCCC TCA TAG TTA GCG TAA CG-3' (-96 glll sequencing primer, provided in a C7C kit, New England Biolabs). The amino acid sequences of displayed peptides were then deduced from the DNA sequence. The three peptide sequences with highest frequency (CTKPDKGYC, CEGHSHRHC and CYGPNNHIC) were identified and sequentially named as NF-1, NF-2 and NF-3 for further experiments.

Enriching comparison of selected phage clones in tumour and control organs

Phages NF-1, NF-2 and NF-3 $\left(1 \times 10^{11}\right.$ PFU in $\left.200 \mu \mathrm{lPBS}\right)$ were respectively injected into the tail veins of anaesthetised mice ( $n=6$ per group). The number of phages bounding to osteosarcoma vasculature and control organs (heart, brain, lung, liver and kidney) were determined and compared.

\section{Immunohistochemical staining of the phage NF-1}

The phage NF-1 $\left(1 \times 10^{11}\right.$ PFU in $200 \mu$ PBS) was injected into the tail veins of anaesthetised mice. After 10 minutes, the mice were perfused via the left ventricle with $20 \mathrm{ml}$ of DMEM. The inferior vena cava was cut for the outlet. Tumours and control organs (heart, brain, lung, liver and kidney) were harvested and fixed with formalin and embedded in paraffin. The paraffin-embedded specimens were cut into $4 \mathrm{~mm}$ sections and baked for $1 \mathrm{~h}$ at $65^{\circ} \mathrm{C}$. All sections were deparaffinised with xylenes and rehydrated through a graded ethanol series into distilled water. Then, the sections were submerged into EDTA antigenic retrieval buffer ( $\mathrm{pH}$ 8.0) and microwaved for antigenic retrieval. The sections were blocked with $0.3 \%$ $\mathrm{H}_{2} \mathrm{O}_{2}$ (3\% in PBS) at room temperature for 510 minutes. After being washed by PBS for $5 \mathrm{~min}$ at $37^{\circ} \mathrm{C}$, the sections were incubated with normal sheep serum for 20 minutes at $37^{\circ} \mathrm{C}$. Subsequently, sections were incubated overnight at $4^{\circ} \mathrm{C}$ with mouse anti-M13 phage antibody with a work dilution of $1: 1000$. The next day, the sections were rinsed three times (10 minutes for each rinse) in PBS and incubated with secondary antibody for 1 hour at room temperature. Subsequntly, the sections were rinsed three times ( 5 minutes for each rinse) in PBS. The bound antibody was visualised using DAB. Sections were rinsed three times ( 5 minutes for each rinse) in running tap water before staining by haematoxylin and eosin. Finally, sections were rinsed for 10 minutes in running tap water before dehydration and mounting. The samples were 
examined with a BX50 microscope (Olympus, Tokyo, Japan) equipped with an AxioVision system (Carl-Zeiss, Germany).

\section{Synthesis and conjugation of peptide NF-1}

The NF-1 peptide, containing nine amino acids with a disulphide bridge connecting the two terminal cysteines was synthesised by Bai Ao Tai Biotech Corp (Guangzhou, China) using standard FMOC chemistry in a solid-phase synthesiser. Fluorescein (FITC)-conjugated peptide (FITCNF-1) contained a spacer of two glycines at the amino-terminus of the cysteine.

\section{In vivo distribution analysis of FITC-NF-1}

FITC-NF-1 was injected intravenously into BALB-c mice bearing osteosarcoma. After 4 hours of circulation, mice were perfused with PBS and then with 4\% paraformaldehyde before their tumours and control organs were obtained, fixed and prepared for scanning by LSCM (Leica, Germany).

\section{Statistical analysis}

Statistical analyses were performed with SPSS 16.0 for Windows. Means were compared using one-way analysis of variance (ANOVA); $p$ values $<0.05$ were considered statistically significant.

\section{Results}

\section{Enrichment of phages homing to osteosarcoma} vasculature

After the four rounds of in vivo selection, the number of phages recovered from each gram of tumour was increased for 1.76 folds (from $4.5 \times 10^{7} \mathrm{PFU} / \mathrm{g}$ to $7.9 \times 10^{7} \mathrm{PFU} / \mathrm{g}$ ), while there was a decrease in the number of phages recovered from each gram of control organs (heart, brain, lung, liver and kidney) as shown in Fig. 1. In order to further clear the phages that bound to the normal vasculature and other non-tumour antigens, we re-injected $1.0 \times 10^{11} \mathrm{PFU}$ of the eluate from the tumour of the last cycle into the tail vein of normal non-tumour-bearing BALB-c mice and recovered 3.3 $\times 10^{10}$ PFU from serum after 10 minutes of circulation.

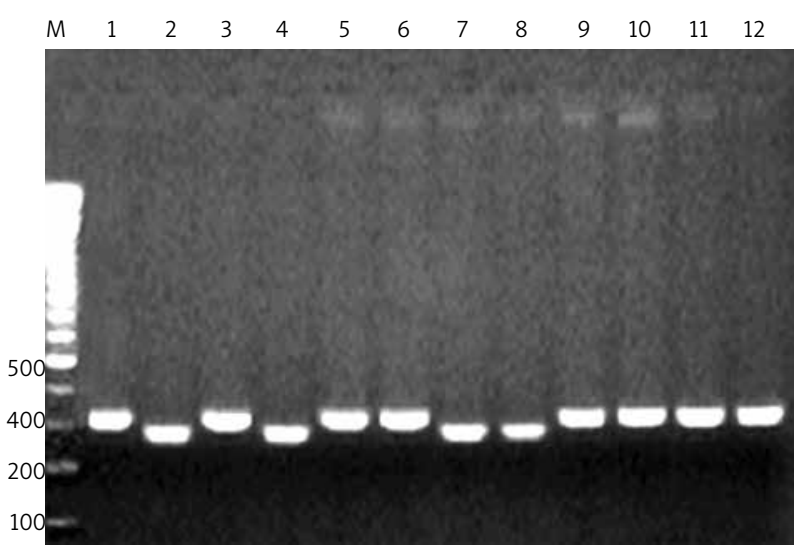

Fig. 2. Elimination of the phages without inserted sequences. DNA clones without inserted sequences with higher speed could be seen in lane numbers 2, 4, 7 and 8
Elimination of the phages without inserted sequences and sequence analysis

Using the method described in Materials and methods herein, 11 clones without inserted sequences were identified and eliminated from the 72 clones (Fig. 2). The other 61 individual clones with inserted sequences were sequenced last. The amino acid sequences of the displayed peptides were then deduced from the DNA sequences of the phages. The three peptide sequences with the highest frequency of occurrence were identified: NF-1 (23 times, $37.7 \%$ ), NF-2 (12 times, 19.7\%) and NF-3 (4 times, 6.6\%).

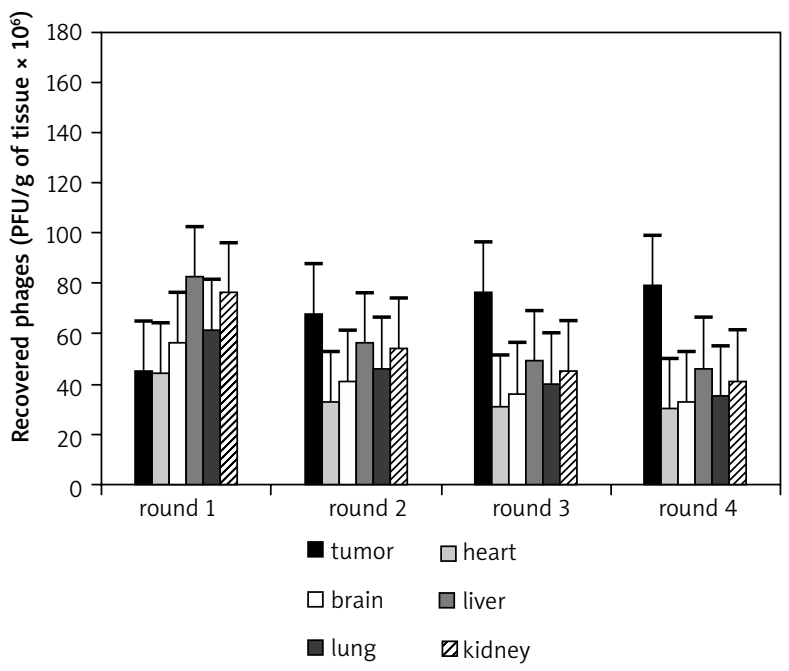

Fig. 1. Recovery of phages from the tumour and control organs during the four in vivo screening rounds. The library $\left(1 \times 10^{11}\right.$ PFU $)$ was injected into the tail vein of the tumour-bearing mice. After four rounds of screening, the phages recovered from each gram of tumour increased from $4.5 \times 10^{7} \mathrm{PFU} / g$ to $7.9 \times 10^{7} \mathrm{PFU} / \mathrm{g}$, while there was a decrease in the number of phages recovered from each gram of control organs

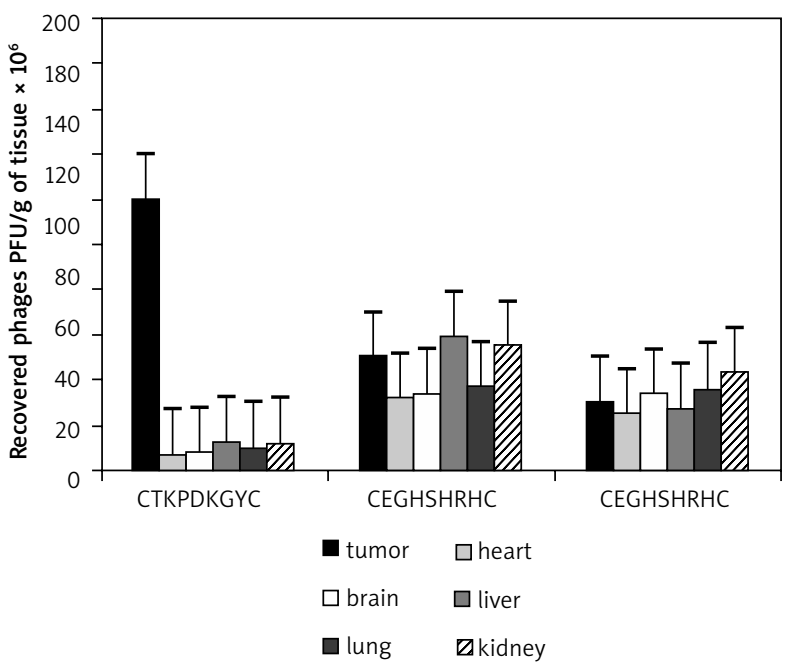

Fig. 3. Phages NF-1, NF-2 and NF-3 were respectively re-injected into the tail veins of the tumour-bearing mice ( $n=6$ per group). The amount of phage NF-1 recovered from the tumour was significantly higher than that from control organs $(p<0.01)$. No statistical difference was detected in the amount of phage NF-2 and NF-3 recovered from the tumour and control organs 

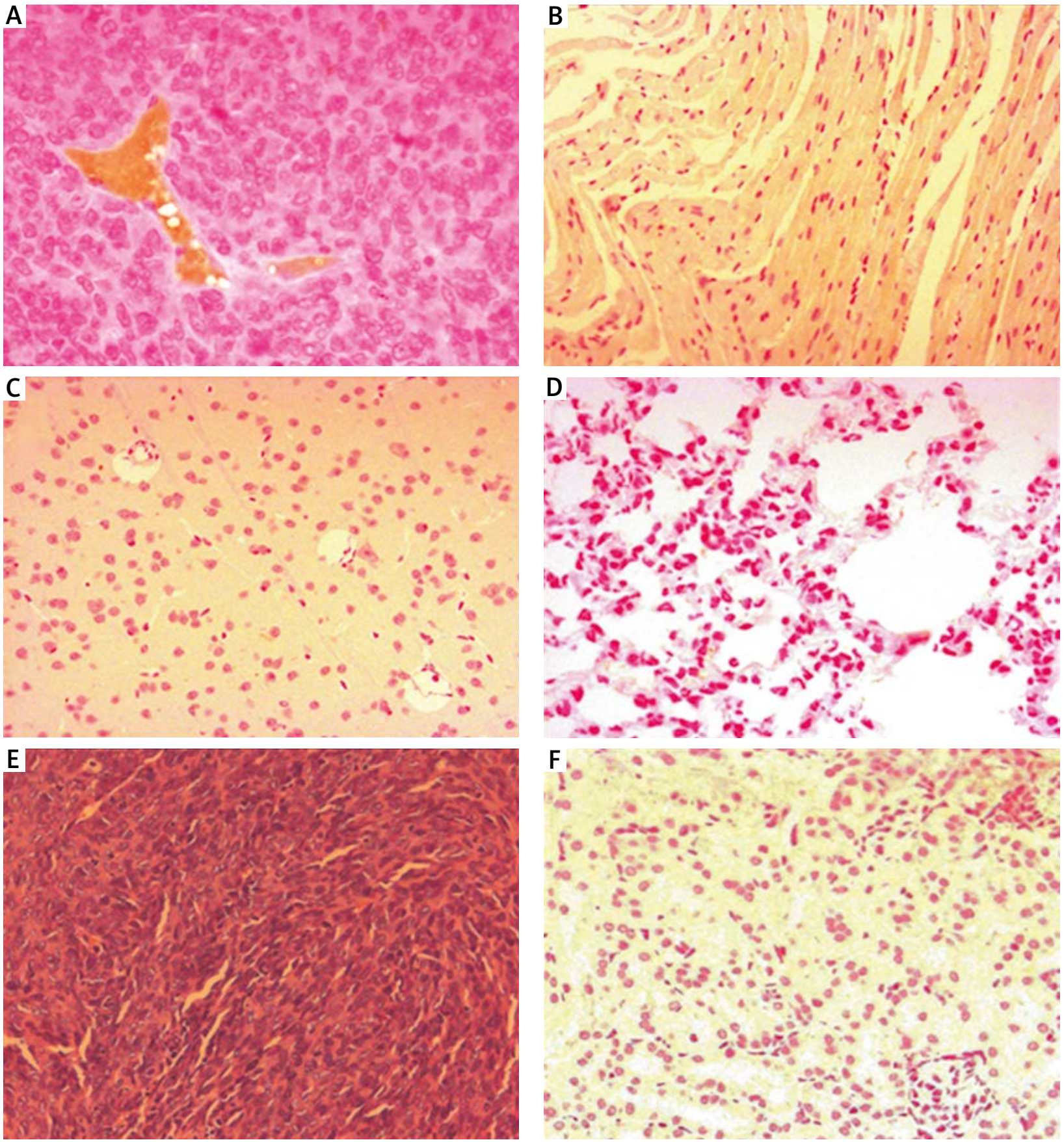

Fig. 4. Immunohistochemical staining of phage NF-1 after intravenous injection into tumour-bearing mice (magnification 200x). Obvious phage stains (yellow brown) could be seen in tumour vessels (A) but was almost undetectable in heart (B), brain (C), lung (D), liver (E) and kidney (F)

In vivo validation of phage clones NF-1, NF-2 and NF-3

We validated the three phages (NF-1, NF-2 and NF-3) with the highest frequency of occurrence by re-injecting them into the tail veins of the mice baring osteosarcoma ( $n=6$ per group). The phage NF-1 recovered from the tumours was $1.1 \times 10^{8} \mathrm{PFU} / \mathrm{g}$, which was 15.7 times higher than the amount recovered from heart $\left(7.0 \times 10^{6} \mathrm{PFU} / \mathrm{g}\right)$, 13.8 times higher than brain $\left(8.0 \times 10^{6} \mathrm{PFU} / \mathrm{g}\right), 8.5$ times higher than liver $\left(1.3 \times 10^{7} \mathrm{PFU} / \mathrm{g}\right), 11.0$ times higher than lung $\left(1.0 \times 10^{6} \mathrm{PFU} / \mathrm{g}\right)$ and 9.2 times higher than kidney $\left(1.2 \times 10^{7} \mathrm{PFU} / \mathrm{g}\right)$. No statistical differences were detected in the amount of phages NF-2 and NF-3 recovered from the tumour and control organs (Fig. 3). Additionally, the phage NF-1 showed abundant binding to the tumour vessel while being much less bonded to the control organs, as shown in immunohistochemical staining (Fig. 4).

\section{In vivo verification of peptide FITC-NF-1}

LSCM showed that FITC signals could be seen in single tumour vessels (Fig. 5A) and tumour vascular plexus (Fig. 5B) but were not detectable in heart (Fig. 5C), brain (Fig. 5D), 

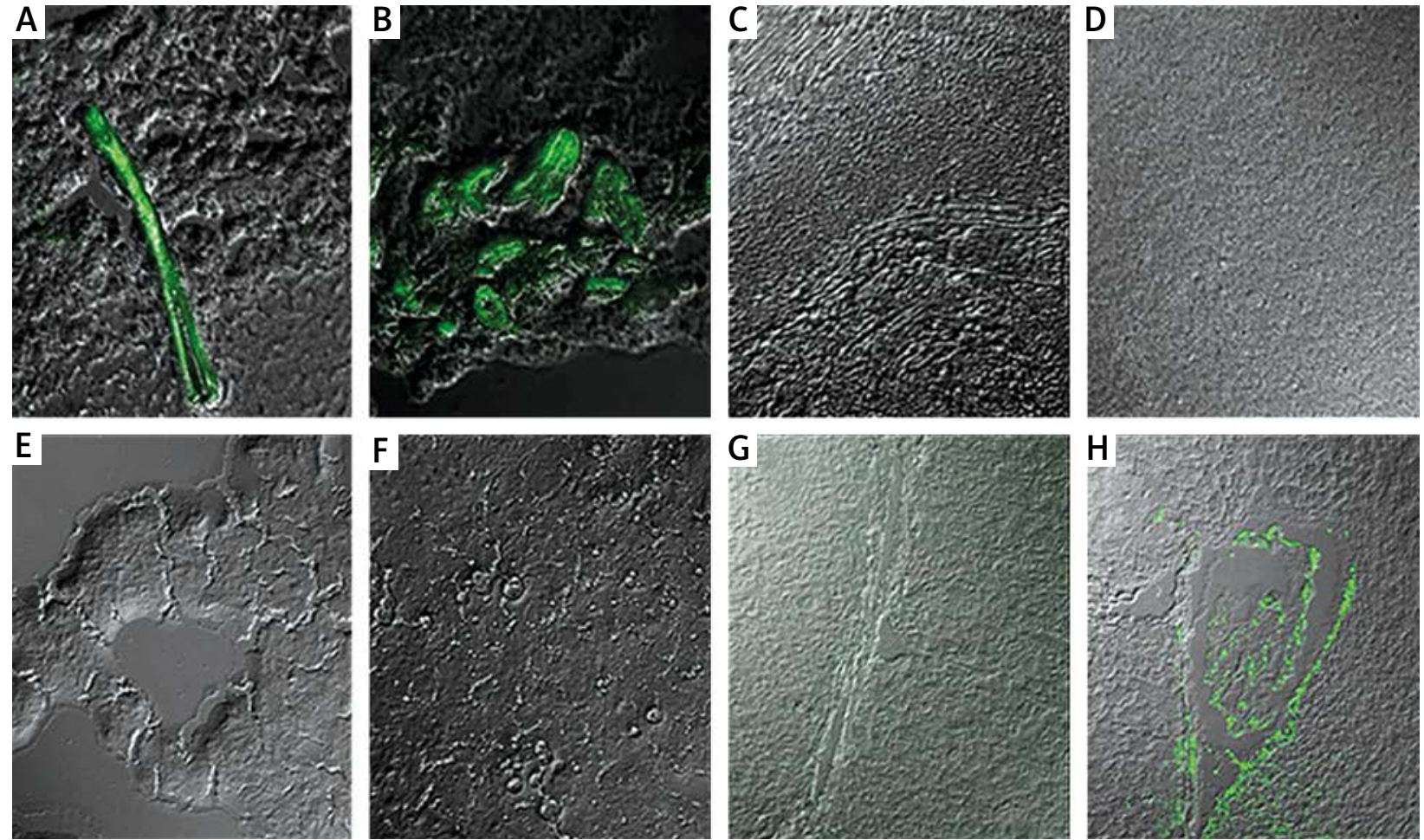

Fig. 5. In vivo banding specificity of FITC-NF-1 peptide to osteosarcoma vasculature confirmed under LSCM (magnification 600x). Obvious FITC signals (green) could be seen in single tumour vessel (A) and tumour vascular plexus (B) but was undetectable in heart (C), brain (D), liver (E) and lung $(\mathbf{F})$. The signals could also be detected in renal glomerulus and renal tubule (H) but not in renal vascular endothelium (G)

liver (Fig. 5E) and lung (Fig. 5F). The signals could also be detected in renal glomerulus and renal tubule (Fig. 5H) but not in renal vascular endothelium (Fig. 5G), suggesting that FITC-NF-1 might be excreted mainly through the renal-urinary route.

\section{Discussion}

Novel peptides with high specificity to tumour tissue may be used as a promising tool to direct diagnostic or therapeutic agents at the tumour. Screening of phage libraries is one of the most commonly used ways to rapidly identify these tumour-selective peptides. Compared with in vitro phage screening, the in vivo phage screening process is more likely to maintain the native structure and functional conformation of the targeted cells or tissue. Accordingly, peptides obtained by in vivo phage screening may possess a higher potential value in clinical utilisation [23]. Conventional chemotherapy drugs at present are mainly designed to target tumour cells. However, it is often difficult for these drugs to be absorbed by tumour cells because they can seldom accumulate into the tumour mass due to poor blood perfusion, high interstitial pressure and abnormal vasculature inside the tumour mass $[19,23]$. In addition, tumour cells are genetically unstable and often produce resistance to multiple chemotherapeutic drugs [24]. In contrast with tumour cells, tumour endothelial cells have good genetic stability, so they rarely produce drug resistance. Tumour blood vessels are also highly accessible to any intravenously administered agents [25] Therefore, we performed in vivo phage screening using osteosarcoma vasculature as the target in this study. We used Ph.D.-C7C library since it displays a random 7-residue peptide that is conformation restrained by the disulphide bond formed by the two cysteines on the two ends. This circular structure is thought to be more stable and natural than a linear structure [26]. For in vivo selection, circulation time of the phages is important. Phages need enough time to bind their target. However, if the circulation time is too long, they will be then taken up by the cells. A duration of 5-15 minutes is thought to be suitable for the phages to bind on the endothelial cells in the blood vessels while not being taken by the cells [27]. In this study, we kept the phages in circulation for 10 minutes. In order to clear phages that bound to the normal vasculature and other non-tumour antigens, we committed a subtractive screening with eluate of the last cycle from the tumour in normal non-tumour-bearing BALB-c mice. There is a certain percentage of phage clones without inserted sequences in the phage library. It would be wasteful and even misleading if this kind of clone were chosen to be sequenced. Therefore, we identified and eliminated them by agarose gel electrophoresis. Finally, a total of 61 clones with inserted sequences were sent to be sequenced, and phage NF-1, NF-2 and NF-3 were found to be most enriched of all sequenced clones. When the three phage clones were re-injected into the tail veins of the tumour-baring mice, respectively, phage NF-1 demonstrated high tumour vasculature-binding specificity confirmed by the much higher amount of phages recovered from tumours than from that recovered from control organs. The amount of phage NF-2 and NF-3 recovered 
from the tumour was not obviously higher than that from the control organs. Binding specificity of phage NF-1 was further confirmed by immunohistochemical staining. We then synthesised and conjugated peptide NF-1 with FITC. The in vivo binding specificity to tumour vasculature of peptide FITC-NF-1 was confirmed under LSCM. The fluorescence could also be detected in renal glomerulus and renal tubule but not in renal vascular endothelium, indicating that peptide FITC-NF-1 may be mainly excreted through the renal-urinary route [22].

In future research, we would like to attempt to conjugate NF-1 with certain drugs and explore the efficacy of targeted therapy for osteosarcoma. In order to explore the diagnostic value for osteosarcoma, we would also like to try to radiolabel peptide NF-1 to examine its effect in tumour imaging [28]. In addition, the receptor of peptide NF-1 on tumour vasculature could also be identified and analysed [29].

In conclusion, the novel 7-residue peptide NF-1 demonstrated obvious binding specificity to osteosarcoma vasculature and may have the potential value to be used in the diagnosis and treatment of osteosarcoma.

The authors declare no conflict of interest.

This work was supported by the National Natural Science Foundation of China (No: 30371450) and by Guangdong Natural Science Foundation (No: S2012010009184).

\section{References}

1. Broadhead ML, Clark JC, Myers DE, Dass CR, Choong PF. The mo lecular pathogenesis of osteosarcoma: a review. Sarcoma 2011; 2011: 959248.

2. Longhi A, Errani C, De Paolis M, Mercuri M, Bacci G. Primary bone osteosarcoma in the pediatric age: state of the art. Cancer Treat Rev 2006; 32: 423-36.

3. Chou AJ, Gorlick R. Chemotherapy resistance in osteosarcoma: current challenges and future directions. Expert Rev Anticancer Ther 2006; 6: 1075-85.

4. Bacci G, Forni C, Ferrari S, et al. Neoadjuvant chemotherapy for osteosarcoma of the extremity: intensification of preoperative treatment does not increase the rate of good histologic response to the primary tumor or improve the final outcome. J Pediatr Hematol Oncol 2003; 25: 845-53.

5. Scotlandi K, Picci P, Kovar H. Targeted therapies in bone sarcomas. Curr Cancer Drug Targets 2009; 9: 843-53.

6. Nyberg P, Salo T, Kalluri R. Tumor microenvironment and angio genesis. Front Biosci 2008; 13: 6537-53.

7. Cao Y, Arbiser J, D'Amato RJ, et al. Forty-year journey of angiogenesis translational research. Sci Transl Med 2011; 3: 113r-4r.

8. St CB, Rago C, Velculescu V, et al. Genes expressed in human tumor endothelium. Science 2000; 289: 1197-202.

9. Kopczyńska E, Makarewicz R. Endoglin - a marker of vascular en dothelial cell proliferation in cancer. Contemp Oncol (Pozn) 2012; 16: 68-71.

10. Sawada J, Urakami T, Li F, et al. Small GTPase R-Ras regulates integrity and functionality of tumor blood vessels. Cancer Cell 2012; 22: 235-49.

11. Białas M, Dyduch G, Adamek D. The tumour and its microenvironment - a complicated interplay. Contemp Oncol (Pozn) 2011; 15: 305-8.

12. Brzozowa M, Wojnicz R, Kowalczyk-Ziomek G, Helewski K. The Notch ligand Delta-like 4 (DLL4) as a target in angiogenesis-based cancer therapy? Wspolczesna Onkol 2013; 17: 234-7.
13. Bussolati B, Grange C, Tei L, Deregibus MC, Ercolani M, Aime S, Camussi G. Targeting of human renal tumor-derived endothelial cells with peptides obtained by phage display. J Mol Med (Berl) 2007; 85: 897-906.

14. Gultepe E, Reynoso FJ, Jhaveri A, et al. Monitoring of magnetic targeting to tumor vasculature through MRI and biodistribution. Nanomedicine (Lond) 2010; 5: 1173-82.

15. Roth P, Hammer C, Piguet AC, Ledermann M, Dufour JF, Waelti E. Effects on hepatocellular carcinoma of doxorubicin-loaded immunoliposomes designed to target the VEGFR-2. J Drug Target 2007; 15: 623-31.

16. Deutscher SL. Phage display in molecular imaging and diagnosis of cancer. Chem Rev 2010; 110: 3196-211.

17. Lee S, Xie J, Chen X. Peptide-based probes for targeted molecular imaging. Biochemistry 2010; 49: 1364-76.

18. Li ZJ, Cho CH. Development of peptides as potential drugs for cancer therapy. Curr Pharm Des 2010; 16: 1180-9.

19. Chen K, Yap LP, Park R, Hui X, Wu K, Fan D, Chen X, Conti PS. A Cy5.5-labeled phage-displayed peptide probe for near-infrared fluorescence imaging of tumor vasculature in living mice. Amino Acids 2012; 42: 1329-37.

20. Li ZJ, Wu WK, Ng SS, et al. A novel peptide specifically targeting the vasculature of orthotopic colorectal cancer for imaging detection and drug delivery. J Control Release 2010; 148: 292-302.

21. Lee NK, Kim M, Choi JH, Kim EB, Lee HG, Kang SK, Choi YJ. Identification of a peptide sequence targeting mammary vasculature via RPLPO during lactation. Peptides 2010; 31: 2247-54.

22. Sun X, Niu G, Yan Y, et al. Phage display-derived peptides for osteosarcoma imaging. Clin Cancer Res 2010; 16: 4268-77.

23. Trepel M, Arap W, Pasqualini R. In vivo phage display and vascular heterogeneity: implications for targeted medicine. Curr Opin Chem Biol 2002; 6: 399-404.

24. Ullah MF. Cancer multidrug resistance (MDR): a major impediment to effective chemotherapy. Asian Pac J Cancer Prev 2008; 9: 1-6.

25. Ruoslahti E, Bhatia SN, Sailor MJ. Targeting of drugs and nanoparticles to tumors. J Cell Biol 2010; 188: 759-68.

26. Fani M, Psimadas D, Zikos C, Xanthopoulos S, Loudos GK, Bouziotis $P$, Varvarigou AD. Comparative evaluation of linear and cyclic $99 \mathrm{mTC}-\mathrm{RGD}$ peptides for targeting of integrins in tumor angiogenesis. Anticancer Res 2006; 26: 431-4.

27. Li ZJ, Cho CH. Peptides as targeting probes against tumor vasculature for diagnosis and drug delivery. J Transl Med 2012; 10 Suppl 1: S1.

28. Kinsella JM, Jimenez RE, Karmali PP, Rush AM, Kotamraju VR, Gianneschi NC, Ruoslahti E, Stupack D et al. X-ray computed tomography imaging of breast cancer by using targeted peptide-labeled bismuth sulfide nanoparticles. Angew Chem Int Ed Engl 2011; 50: 12308-11.

29. Teesalu T, Sugahara KN, Ruoslahti E. Mapping of vascular ZIP codes by phage display. Methods Enzymol 2012; 503: 35-56.

\section{Address for correspondence \\ Zhanjun Shi}

Department of Orthopaedic Surgery

Nanfang Hospital

Southern Medical University

Guangzhou, Guangdong Province, 510515, China

tel./fax +86 2062787191

e-mail: hipknee66@163.com

Submitted: 8.10 .2013

Accepted: $\quad 30.10 .2013$ 